\title{
Adaptações do sistema respiratório referentes à função pulmonar em resposta a um programa de alongamento muscular pelo método de Reeducação Postural Global Respiratory system adaptations relative to pulmonary function in response to a muscle stretching program using the Global Posture Reeducation method
}

\author{
Marlene Aparecida Moreno', Aparecida Maria Catai ${ }^{2}$, Rosana Macher Teodori ${ }^{3}$, \\ Bruno Luis Amoroso Borges ${ }^{4}$, Roberta Silva Zuttin ${ }^{5}$, Ester da Silva ${ }^{6}$
}

Estudo desenvolvido no

Laboratório de Pesquisa em

Fisioterapia Cardiovascular e de Provas Funcionais do PPG-Ft Programa de Pós-Graduação em Fisioterapia da Unimep Universidade Metodista de Piracicaba, Piracicaba, SP, Brasil

1 Fisioterapeuta; Profa. Dra. do PPG-Ft da Unimep

2 Fisioterapeuta; Profa. Dra. do PPG-Ft da Universidade Federal de São Carlos, São Carlos, SP

3 Fisioterapeuta; Profa. Dra. do PPG-Ft da Unimep

4 Fisioterapeuta; Prof. Ms. do Curso de Fisioterapia da Unimep

5 Fisioterapeuta Ms.

6 Fisioterapeuta; Profa. Dra. do PPG-Ft da Unimep

Colaborador: Marcelo de Castro Cesar, Prof. Dr. do PPG em Educação Física da Unimep

\section{ENDEREÇO PARA}

CORRESPONDÊNCIA

Marlene A. Moreno

R. Santa Cruz 990 Bairro Alto 13419-030 Piracicaba SP

e-mail:

ma.moreno@terra.com.br

Apoio financeiro: Fundo de Apoio à Pesquisa da Unimep

ApresentaÇÃo

out. 2008

ACEITO PARA PUBLICAÇÃO

jan. 2009
Resumo: A proposta deste estudo foi analisar as adaptações do sistema respiratório referentes à função pulmonar em resposta ao alongamento da cadeia muscular respiratória pelo método de Reeducação Postural Global (RPG). Foram estudados 20 homens, sedentários, de antropometria semelhante, sem antecedentes de doenças musculoesqueléticas ou cardiorrespiratórias. Os voluntários foram divididos aleatoriamente em dois grupos de dez, sendo um grupo controle (GC), que não participou do protocolo de alongamento, e o outro submetido à intervenção pelo método de RPG, denominado grupo tratado (GT). O alongamento foi realizado duas vezes por semana, durante oito semanas, totalizando 16 sessões. Os dois grupos foram submetidos à prova de função pulmonar, medindo-se a capacidade vital lenta, capacidade vital forçada e ventilação voluntária máxima, antes e após o período de intervenção. Os valores obtidos em todas as variáveis dos voluntários do GC na avaliação inicial não apresentaram diferença estatisticamente significante quando comparados aos obtidos na avaliação final $(p>0,05)$. No GT os valores finais apresentaram-se significativamente maiores que os iniciais $(p<0,05)$. O protocolo de alongamento da cadeia muscular respiratória proposto pelo método de RPG mostrou pois ser eficiente para promover aumento das variáveis espirométricas, sugerindo que pode ser utilizado como um recurso fisioterapêutico coadjuvante às condutas de fisioterapia respiratória.

Descritores: Exercícios de alongamento muscular; Exercícios respiratórios; Postura

ABSTRACT: The aim of this study was to analyse the respiratory system adaptations concerning pulmonary function in response to stretching the respiratory muscle chain, by means of Global Posture Reeducation (GPR). Twenty sedentary young men with similar anthropometry and no history of musculoskeletal or cardiorespiratory disease were randomly divided into two groups of ten each: control group (CG), who did no stretching, and treated group (TG), submitted to GPR. Stretching was carried out twice a week for 8 weeks, in a total of 16 sessions. The two groups were submitted to pulmonary function tests to assess slow vital capacity, forced vital capacity and maximal voluntary ventilation, before and after the intervention period. The initial values of all spirometric variables measured in CG volunteers showed no statistically significant differences when compared to those of the final evaluation ( $p>0.05)$, whereas in TG all values increased after intervention $(p<0.05)$. The GPR respiratory muscle chain stretching protocol thus proved efficient to promote an increase in spirometric variables, suggesting that it may be used as an auxiliary resource in respiratory physical therapy.

KEY words: Breathing exercises; Muscle stretching exercises; Posture 


\section{INTRODUÇÃO}

A relação entre atividade física e saúde está bem estabelecida: os exercícios físicos associam-se a um estilo de vida saudável e aumento da expectativa de vida e, cada vez mais, mostram indícios de efeitos benéficos à saúde.

Alguns autores estudaram o comportamento da função pulmonar em sujeitos saudáveis frente à realização de treinamentos físicos diversos ${ }^{1,2}$ e os resultados demonstram que, mesmo saudáveis, os sedentários apresentam pior função pulmonar quando comparados a sujeitos ativos ${ }^{3,4}$. Como um dos efeitos da inatividade, a mobilidade muscular pode ser alterada devido às modificações das proteínas contráteis e do metabolismo das mitocôndrias, resultando em atrofia, fraqueza ${ }^{5}$, diminuição do número de sarcômeros e aumento na deposição de tecido conjuntivo ${ }^{6}$, levando ao encurtamento muscular e limitação da mobilidade articular.

Apesar de a musculatura respiratória não ser passível de imobilização, sua constante ação de contração favorece uma postura em inspiração; o encurtamento dos músculos da cadeia respiratória leva ao aumento do volume pulmonar em repouso, caracterizando tórax com grande diâmetro ${ }^{7}$, restringindo assim a mobilidade da caixa torácica. Portanto, para que ocorram os ciclos respiratórios de forma adequada, deve haver um sincronismo entre a caixa torácica, os pulmões e a musculatura respiratória, atuando de forma harmoniosa e coordenada para tornar possível um fenômeno tão complexo ${ }^{8}$.

O exercício de alongamento muscular tem sido bastante estudado e difundido, pois permite que o músculo recupere seu comprimento necessário para manter o alinhamento postural correto e a estabilidade articular, garantindo principalmente a integridade e a função muscular ${ }^{9,10}$. No entanto, estudos sobre seu efeito na função pulmonar ainda são incipientes, possivelmente por se tratar de um grupo muscular de funcionamento complexo. O método de Reeducação Postural Global (RPG), baseado nas cadeias musculares posturais, apresenta preocupação especial com o alongamento da musculatura respiratória e tem demonstrado influência positiva na mecânica respiratória ${ }^{11}$. Considerando a proposta do método de RPG, este estudo visou analisar, em homens sedentários, as adaptações do sistema respiratório referentes à função pulmonar em resposta ao alongamento da cadeia muscular respiratória, na postura da RPG "rã no chão com os braços abertos".

\section{METODOLOGIA}

Este é um estudo prospectivo, aprovado pelo Comitê de Ética em Pesquisa da Unimep. Os voluntários foram informados e esclarecidos a respeito dos objetivos e da metodologia experimental à qual seriam submetidos, explicitando o caráter não-invasivo dos procedimentos. Todos assinaram o termo de consentimento livre e esclarecido.

O cálculo amostral foi feito usando o aplicativo GraphPad StatMate (v. 1.01i) para o nível de confiança de $95 \%$ e poder de força da amostra (power) de $80 \%$, utilizando-se a variável CVF. Participaram do estudo 20 voluntários jovens, estudantes do Curso de Fisioterapia da Unimep, do sexo masculino, nãotabagistas, de antropometria semelhante e sedentários, ou seja, com capacidade aeróbia "fraca", de acordo com a classificação da American Heart Association $^{12}$. Esta foi avaliada pelo teste ergoespirométrico realizado em cicloergômetro Corival 400 (Quinton, Seattle, WA, USA), utilizando um analisador metabólico específico (CPXD, Med Graphics, St. Paul, MN, USA). Apesar de hígidos, todos apresentavam força muscular inspiratória abaixo do predito, de acordo com a equação de predição dos valores normais proposta por Neder et al. ${ }^{13}$, segundo os as medidas obtidas das pressões inspiratórias máximas $\left(\mathrm{PI}_{\text {máx }}\right)$ (Tabela 1). Não tinham antecedentes de doenças musculoesqueléticas, cardiovasculares ou respiratórias, conforme avaliação clínica prévia, e apresentavam encurtamento da cadeia muscular respiratória, de acordo com avaliação postural preconizada por Souchard e Ollier ${ }^{14}$.

Os voluntários foram divididos em dois grupos de dez por randomização realizada por tabela numérica, sendo um grupo controle (GC), que não participou do protocolo de alongamento, e o outro submetido à intervenção pelo método de RPG, denominado grupo tratado (GT).

Antes e após o período de intervenção, todos os voluntários foram submetidos à avaliação da função pulmonar por espirometria. A amostra foi familiarizada com todos os procedimentos antes do início do experimento; durante o período experimental não houve perda amostral. O pesquisador que realizou todas as medidas era cegado, ou seja, não tinha conhecimento se o voluntário era do GC ou GT, para que não houvesse influência nos resultados. Todos os experimentos foram realizados no mesmo período do dia (entre as 14 h e 17 h), com o objetivo de evitar as influências do ciclo circadiano nas variáveis estudadas.

O voluntário repousou por 10 minutos antes do teste e todos os procedimentos Ihe foram descritos cuidadosamente. As provas de função pulmonar foram realizadas de acordo com as orientações da American Thoracic Society ${ }^{15}$ e das

Tabela 1 Características (média \pm desvio padrão) dos grupos controle (GC) e tratado (GT)

\begin{tabular}{lrr}
\hline Característica & GC $(\mathrm{n}=10)$ & GT $(\mathrm{n}=10)$ \\
\hline Idade (anos) & $23,4 \pm 2,7$ & $22,9 \pm 2,0$ \\
Massa corporal $(\mathrm{kg})$ & $81,1 \pm 7,3$ & $80,0 \pm 4,3$ \\
Estatura $(\mathrm{cm})$ & $177,7 \pm 6,1$ & $176,4 \pm 5,7$ \\
IMC $\left(\mathrm{kg} / \mathrm{m}^{2}\right)$ & $25,6 \pm 1,2$ & $25,1 \pm 1,9$ \\
PImáx predita $\left(\mathrm{cmH}_{2} \mathrm{O}\right)$ & $-139,4 \pm 3,8$ & $-139,09 \pm 2,4$ \\
PImáx obtida $\left(\mathrm{cmH}_{2} \mathrm{O}\right)$ & $-104,5 \pm 12,1$ & $-105,5 \pm 11,1$ \\
VO 2 pico $\left(\mathrm{mL} / \mathrm{kg} / \mathrm{min}^{-1}\right)$ & $30,3 \pm 4,6$ & $30,2 \pm 4,1$ \\
\hline
\end{tabular}

$\mathrm{IMC}=$ Índice de massa corporal; $\mathrm{PI}_{\text {máx }}$ : pressão inspiratória máxima; $\mathrm{VO}_{2 \text { pico }}=$ consumo de oxigênio no pico do exercício (não houve diferença estatisticamente significante em qualquer das variáveis, $p>0,05$ ) 
diretrizes para testes de função pulmonar de Pereira ${ }^{16}$, usando um espirômetro (Med Graphics Breeze 6.0, St. Paul, MN, USA). Nessas provas foram medidas a capacidade vital lenta (CVL), capacidade vital forçada (CVF) e ventilação voluntária máxima (VVM). Cada manobra foi realizada até se obterem três curvas aceitáveis e duas reprodutíveis, não excedendo mais que oito tentativas. Os valores de referência utilizados foram os de Pereira ${ }^{16}$, e os resultados obtidos, expressos em condições de temperatura corporal e pressão ambiente saturada com vapor d'água (BTPS body temperature and pressure saturated).

O exame foi realizado com o voluntário sentado, a cabeça mantida em posição neutra e fixa, e um clipe nasal foi usado para evitar vazamento de ar pelas narinas. Pelos testes espirométricos foram obtidos os valores absolutos e em porcentagem do previsto para cada grupo, referentes à $C V L$, capacidade inspiratória $(\mathrm{Cl}), \mathrm{CVF}$, volume expiratório forçado no primeiro segundo $\left(\mathrm{VEF}_{1}\right)$ eVVM.

As cadeias musculares submetidas ao alongamento utilizando a postura "rã no chão com braços abertos" foram: a) cadeia inspiratória, constituída pelos músculos escalenos, peitoral menor, intercostais e diafragma e seu tendão; b) cadeia ântero-interna do ombro, constituída pelos músculos subescapular, coracobraquial e peitoral maior; c) cadeia anterior do braço, constituída pelos músculos trapézio superior, deltóide médio, coracobraquial, bíceps braquial, braquiorradial, pronador redondo, palmares, flexores dos dedos, tenares e hipotenares; d) cadeia ântero-interna do quadril, constituída pelos músculos psoas ilíaco, pectíneo, adutor curto, adutor longo, grácil e porção anterior do adutor magno ${ }^{17}$.

Previamente à realização da postura, os voluntários do GT foram submetidos à manobra para relaxamento do diafragma, preparando-o para o alongamento ${ }^{7}$. Esta consistiu de uma massagem realizada bilateralmente com a ponta dos dedos, aplicada desde o ângulo costoxifoidiano até as últimas costelas, utilizando pressões suaves sobre a pele. Para a postura "rã no chão com os braços abertos", o voluntário foi posicionado em decúbito dorsal com os braços a

Tabela 2 Medidas espirométricas (valores absolutos e percentual em relação aos preditos, \%) antes e após o tratamento nos grupos controle (GC) e tratado (GT)

\begin{tabular}{lrrrr}
\hline $\begin{array}{l}\text { Variáveis } \\
\text { espirométricas }\end{array}$ & \multicolumn{2}{c}{ Antes $(\mathrm{n}=10)$} & \multicolumn{2}{c}{ GT $(\mathrm{n}=10)$} \\
\hline CVL (L) & $5,42 \pm 0,4$ & $5,25 \pm 0,6$ & $4,98 \pm 1,0$ & $5,38 \pm 1,0^{*}$ \\
CVL \% & $97,7 \pm 9,3$ & $94,9 \pm 16,0$ & $92,4 \pm 16,3$ & $97,3 \pm 18,2^{*}$ \\
Cl (L) & $3,64 \pm 0,4$ & $3,72 \pm 0,3$ & $3,41 \pm 0,5$ & $3,76 \pm 0,6^{*}$ \\
Cl \% & $101,2 \pm 12,7$ & $104 \pm 9,3$ & $98,1 \pm 15,7$ & $109 \pm 17,5^{*}$ \\
CVF (L/s) & $5,6 \pm 0,5$ & $5,54 \pm 0,3$ & $5,46 \pm 0,7$ & $5,60 \pm 0,8^{*}$ \\
CVF \% & $100,5 \pm 7,7$ & $99,7 \pm 7,6$ & $100,8 \pm 10,9$ & $104,2 \pm 11,7^{*}$ \\
VEF1 (L/s) & $4,57 \pm 0,3$ & $4,53 \pm 0,3$ & $4,49 \pm 0,5$ & $4,64 \pm 0,3^{*}$ \\
VEF1 \% & $99,1 \pm 7,7$ & $98,6 \pm 8,3$ & $99,7 \pm 9,7$ & $102,4 \pm 8,6^{*}$ \\
VVM (L/min) & $181,9 \pm 21,0$ & $174,6 \pm 15,0$ & $166,4 \pm 22,0$ & $183,9 \pm 26,0^{*}$ \\
VVM \% & $98,1 \pm 14,4$ & $94,4 \pm 11,6$ & $92 \pm 7,4$ & $99,4 \pm 12,1^{*}$ \\
\hline
\end{tabular}

* diferença antes $x$ após significativa $(p<0,05) ; C V L=$ capacidade vital lenta; $\mathrm{Cl}=$ capacidade inspiratória; $\mathrm{CVF}=$ capacidade vital forçada; $\mathrm{VEF}_{1}=$ volume expiratório forçado no primeiro segundo; VVM = ventilação voluntária máxima

aproximadamente $45^{\circ}$ de abdução, antebraços em supinação, com as palmas das mãos voltadas para cima; membros inferiores com abdução, quadril e joeIhos fletidos até a completa aposição das plantas dos pés. Foi realizada a pompagem dorsal, objetivando o alinhamento das curvaturas dorsal e cervical da coluna vertebral, enquanto a pompagem sacral buscou a retificação da coluna lombar. O voluntário foi solicitado a abduzir os quadris a partir da posição inicial, mantendo as plantas dos pés em aposição, alinhadas ao eixo do corpo.

Para a realização da postura, o terapeuta utilizou comandos verbais e contatos manuais, solicitando a manutenção do alinhamento e as correções posturais necessárias, com o objetivo de otimizar o alongamento e impedir compensações. O voluntário foi solicitado a realizar inspirações tranqüilas seguidas de expirações prolongadas, com o máximo rebaixamento possível das costelas e protrusão do abdome, visando o alongamento da cadeia muscular respiratória, enquanto o terapeuta auxiliava na manutenção do crescimento axial.

Durante a realização da postura, os membros superiores deviam seguir em abdução, com alongamento progressivo dos músculos peitorais, até o limite possível para cada voluntário, evitando compensações. Da mesma forma, os membros inferiores em aposição devi- am avançar em sentido caudal, visando principalmente o alongamento do músculo psoas ilíaco, mantendo a curvatura lombar em contato com a superfície de apoio. A mesma postura foi realizada em todas as sessões, durante 30 minutos, sendo que o terapeuta realizava a progressão da mesma até o limite possível para cada voluntário, dentro de cada sessão, favorecendo o alongamento progressivo das cadeias musculares envolvidas na postura durante o tratamento. O alongamento foi realizado duas vezes por semana, durante oito semanas, totalizando 16 sessões.

Para a análise estatística foi utilizado o aplicativo Statistica for Windows (release 6.1 Stat Soft). O teste de Kolmogorov-Smirnov foi usado para verificar a distribuição dos dados, sendo rejeitada a hipótese de normalidade de todas as variáveis. Portanto, foram utilizados testes não-paramétricos, sendo o de Wilcoxon para amostras pareadas e de Mann-Whitney para amostras nãopareadas, com nível de significância $\alpha=5 \%$.

\section{RESULTADOS}

Verifica-se na Tabela 2 que os dados obtidos da espirometria, expressos em valores absolutos e percentualmente em relação ao previsto, obtidos antes e após o período de intervenção no GC, não 
apresentaram diferença estatisticamente significante $(p>0,05)$. Para o GT, observaram-se maiores valores após o treinamento, encontrando-se diferenças significativas em todas as variáveis estudadas $(p<0,05)$.

\section{DISCUSSÃO E CONCLUSÃO}

Os voluntários estudados não apresentavam discrepância em relação às características antropométricas e à idade, as quais foram cuidadosamente observadas para manter a homogeneidade dos grupos, bem como garantir a qualidade da pesquisa, uma vez que as variáveis espirométricas apresentam variações fisiológicas de acordo com o sexo, idade, massa corporal e estatura ${ }^{18}$. Os mesmos apresentavam ainda encurtamento da cadeia muscular respiratória e valores de força muscular inspiratória abaixo do predito, com conseqüente desvantagem da mecânica respiratória, sendo essas alterações geralmente decorrentes de encurtamento excessivo da musculatura inspiratória, tendo como principais causas agressões neuropsíquicas (estresse), aumento do volume da massa visceral, postura inadequada, patologias respiratórias, fraqueza muscular e envelhecimento ${ }^{19}$.

A interação entre o pulmão e a caixa torácica constitui um determinante importante dos volumes pulmonares, podendo acarretar conseqüências relevantes para a troca gasosa pulmonar. Os testes de função pulmonar são bastante precisos para verificar a eficácia de técnicas, servindo como método auxiliar na avaliação respiratória ${ }^{20-22}$, além de fornecerem medidas quantitativas que constituem também indicadores da função neuromuscular ${ }^{23}$.

São poucos na literatura os estudos sobre função pulmonar em sujeitos saudáveis, mas alguns autores demonstram que, mesmo saudáveis, os sedentários apresentam piores resultados quando comparados a sujeitos ativos ${ }^{3,4}$. Até o momento, são escassos estudos que evidenciem melhora do desempenho da função pulmonar em sujeitos submetidos a intervenção fisioterapêutica pelo método de RPG. No entanto, outras práticas de atividade física têm incluído o alongamento com o objetivo de melhorar a função respiratória, como no trabalho realizado por Mandanmohan et al. ${ }^{24}$, que observaram aumento de índices espirométricos após treinamento regular de ioga, semelhante ao resultado obtido neste estudo utilizando a RPG demonstrando assim que o controle da respiração e o alongamento têm efeito benéfico sobre a função pulmonar.

Uma vez que a $V V M$ reflete a capacidade do indivíduo de sustentar um alto nível de ventilação, ou seja, simula um esforço físico extenuante, o aumento nos valores dessa variável, encontrado no GT após o período de intervenção, talvez possa estar relacionado ao aumento do número de sarcômeros em série promovido pelo alongamento ${ }^{19,25}$, melhorando a interação entre os filamentos de actina e miosina e aumentando a capacidade contrátil dos músculos respiratórios, em virtude do aumento do comprimento funcional dos mesmos ${ }^{26}$. Assim, no presente estudo procurou-se aplicar técnicas de alongamento para melhorar a relação comprimento-tensão das fibras musculares, favorecendo o desempenho da bomba respiratória, considerando que quanto mais alongado estiver um músculo dentro do limite de sua capacidade contrátil, maior será sua capacidade de gerar tensão.

Hoje se aceita que existe uma remodelação dos músculos respiratórios em situações de sobrecarga, que consiste tanto em adaptações estruturais (dos sarcômeros) como metabólicas (capacidade oxidativa $)^{27}$, o que pode ter sido responsável pelo aumento da capacidade contrátil da cadeia muscular respira- tória, com conseqüente aumento da CVL e $\mathrm{Cl}$ nos sujeitos do GT. Segundo West ${ }^{28}$ e Leff e Schumacker ${ }^{29}$, a mudança do volume pulmonar está diretamente relacionada com a pressão que o distende, sendo a pressão gerada para essa distensão dependente de uma contração muscular efetiva.

O efeito do alongamento dos músculos respiratórios nas variáveis pulmonares foi investigado em portadores de doença pulmonar obstrutiva crônica; os resultados mostraram que os pacientes apresentaram aumento da mobilidade torácica e do volume corrente e diminuição da dispnéia ${ }^{30}$, bem como melhora na mecânica respiratória após o período de intervenção ${ }^{31}$. Apesar das características dos voluntários e os protocolos utilizados nos dois estudos serem diferentes dos do presente estudo, os resultados são concordantes quanto aos efeitos benéficos proporcionados pelo alongamento muscular sobre a função respiratória.

O aumento da CVF e do VEF ${ }_{1}$ no GT após o período de intervenção justificase possivelmente pelo fato de os voluntários desta pesquisa não apresentarem obstrução ao fluxo aéreo; e, como a contração dos músculos expiratórios é bastante exigida durante a realização da postura "rã no chão com os braços abertos", esta leva a uma melhora na habilidade de coordenar a ação desse grupo muscular, proporcionando maior efetividade na realização da manobra durante os testes de reavaliação.

Em conclusão, os resultados deste estudo mostraram que o alongamento da cadeia muscular respiratória realizado na postura "rã no chão com os braços abertos", do método de RPG, foi eficiente para promover aumento significativo das variáveis espirométricas dos voluntários estudados, sugerindo que pode ser utilizado como recurso fisioterapêutico coadjuvante às condutas de fisioterapia respiratória. 


\section{REFERENCIAS}

1 Valle PHC, Costa D, Jamami M, Oishi J, Baldissera V. Avaliação do treinamento muscular respiratório e do treinamento físico em indivíduos sedentários e atletas. Rev Bras Ativ Fisica Saude. 1997;2(4):27-40.

2 Valle PHC, Winkelmann ER, Kern E, Silva AMV, Marchi $\mathrm{PB}$, Costa D. Efeitos do treinamento e destreinamento da força muscular respiratória em soldados. Ativ Fisica Saude. 2002;7(1):46-54.

3 Hagberg JM, Yerg JE, Seals DR. Pulmonary function in young and older athletes and untrained men. J Appl Physiol. 1988;65(1):101-5.

4 Doherty M, Dimitrou L. Comparison of lung volume in Greek swimmers, land-based athletes, and sedentary controls using allometric scaling. Br J Sports Med. 1997;31(4):337-41.

5 Hall CM, Brody TB. Exercícios terapêuticos: na busca da função. Rio de Janeiro: Guanabara-Koogan; 1996.

6 Williams P. Effect of intermittent stretch on immobilized muscle. Ann Rheum Dis. 1988;47:1014-6.

7 Souchard PE. Respiração. São Paulo: Summus; 1989.

8 Hillman DR, Finucane KE. A model of the respiratory pump. J Appl Physiol. 1987;63(3):951-61.

9 Marques AP, Ferreira EAG, Matsutani LA, Assumpção A, Capela CE, Pereira CAB. Efeito dos exercícios de alongamento na melhora da dor, flexibilidade e qualidade de vida em pacientes com fibromialgia. Fisioter Mov. 2004;17(4):35-41.

10 Rosário JLR, Marques AP, Maluf AS. Aspectos clínicos do alongamento: uma revisão de literatura. Rev Bras Fisioter. 2004;8(1):83-8.

11 Teodori RM, Moreno MA, Fiori Junior JF, Oliveira ACS. Alongamento da musculatura inspiratória por intermédio da reeducação postural global (RPG). Rev Bras Fisioter. 2003;7(1):25-30.

12 American Heart Association. Committee on Exercise. Exercise testing and training of apparently healthy individuals: a handbook for physicians. Dallas; 1972.

13 Neder JA, Andreoni S, Lerario MC, Nery LE. Reference values for lung function tests, II: maximal respiratory pressures and voluntary ventilation. Braz J Med and Biol Res. 1999;32(6):719-27.

14 Souchard PE, Ollier M. As escolioses: seu tratamento fisioterapêutico e ortopédico. São Paulo: Edit. É Realiz.; 2001.

15 American Thoracic Society. Standardization of spirometry 1994 update. Am J Respir Crit Care Med. 1995; 152:1107-36.
16 Pereira CAC. Diretrizes para testes de função pulmonar. J Pneumol. 2002;28 (Suppl 3):1-82.

17 Souchard PE. Reeducação postural global: método do campo fechado. São Paulo: Ícone; 1987.

18 Costa D, Jamami M. Bases fundamentais da espirometria. Rev Bras Fisioter. 2001;5(2):95-102.

19 Shah SB, Peters D, Jordan KA, Milner DJ, Fridén J, Capetanaki Y, et al. Sarcomere number regulation maintained after immobilization in desmin-null mouse skeletal muscle. J Exp Biol. 2001;204:1703-10.

20 Aaron S, Dales RE, Cardinal P. How accurate is spirometry at predicting restrictive pulmonary impairment? Chest. 1999;115(3):869-73.

21 Timothy J, Barreiro DO, Perillo I. An approach to interpreting spirometry. Am Fam Physician. 2004;69(5):1107-14.

22 Pierce R. Spirometry: an essential clinical measurement. Aust Fam Physician. 2005;34(7):535-9.

23 Costa D, Jamami M, Soares LBT, Pinto JM, Oishi J. Função pulmonar em miopatias hereditárias. Rev Bras Fisioter. 1996;1(2):73-7.

24 Mandanmohan UK, Jatiya L, Bhavanani AB. Effect of yoga training on handgrip, respiratory pressures and pulmonary function. Indian J Physiol Pharmacol. 2003;47(4):387-92.

25 Coutinho EL, Gomes ARS, França CN, Salvini TF. The effect of passive stretching on the immobilized soleus muscle fiber morphology. Braz J Med Biol Res. 2004;37(12):1853-61.

26 Lieber RL, Bodine-Fowler SC. Skeletal muscle mechanics: implications for rehabilitation. Phys Ther. 1993;73(12):844-56.

27 Iturri JBG. Función de los músculos respiratorios en la EPOC. Arch Bronconeumol. 2000;36:275-85.

28 West JB. Respiratory physiology: the essentials. Philadelphia: Williams \& Wilkins; 2004.

29 Leff AR, Schumacker PT. Respiratory physiology: basics and applications. Phyladelphia: WB Saunders; 1993.

30 Kakizaki F, Shibuya M, Yamazaki Y, Yamada M, Suzuki $\mathrm{H}$, Homma I. Preliminary report on the effects of respiratory muscle stretch gymnastics on chest wall mobility in patients with chronic obstructive pulmonary disease. Respir Care. 1999;44(4):409-14.

31 Cunha APN, Marinho PEM, Silva TNS, França EET, Amorim C, Galindo-Filho VC, et al. Efeito do alongamento sobre a atividade dos músculos inspiratórios na DPOC. Saude Rev. 2005; 7(17):13-9. 